\title{
利用火箭和卫星研究宇宙空間 苏联“真理报”
}

1957 年 10 月 4 日，苏联发射了第一个人造地球 卫星, 这个历史性的日子开始了征服宇宙的时代。第 一个卫星的重量是 83.6 公斤。

刚过一个月，1957年 11 月 3 日，継第一个卫星 之后, 多发射了第二个人造卫星, 它載有較为复杂的仪 器和試驗动物一一小狗“萊伊卡”。这个卫星重 508.3 公斤。

1958 年 5 月 15 日又发射了第三个人造卫星, 重 量是 1,327 公斤, 它是一个真正的空中科学实驗窒。

1959年 1 月 2 日发射了第一个宇宙火箭, 这是苏

联科学家、設計师、工程师和工人所获得的义一項重大 成就。在距离月球不远时, 火箭就永远脫离了地球, 成为太阳的一个卫星一一第一个人造行星。这項成就 是以苏联火箭技术的成就为基础的。

現在回頋一下各个人造卫星的主望参数。第一个 卫星存了 92 天，第二个你在了 162 天, 第三个将存 在到 1959 年秋季。第一个卫星围繞地球开始的运行 周期为 96.2 分, 第二个一-103.7 分, 第三个一105.95 分。第一个卫星远地点的高度为 950 公里, 第 二个一-1,670 公里，第三个—-1,880 公里。第一 个卫星近地点的高度为 227 公里, 第二个--225 公 里, 第三个一226 公里。

三个苏联卫星的軌道都与赤道平面成 $65^{\circ}$ 的交角。 由于空气的阻力, 各个卫星的軌道不諭在大小或形状 上都逐漸有所改变。軌道越来越縮短，讲日谷接近地 球婊面。因为軌道长軸的长度一遖在縮小，那么根据 开普勒第三定律，卫苼繞地球运行的周期也，就不断在 縮短。运行風期改变的速度是取决于大气对卫星的阻 力。对各个卫星运行周期的改变細加分析, 可以确定 -大气的某些物理参数, 抹可闻明其昼夜和緯度的差 异。

根据卫尾上各种仪表的讀数, 可以研究卫星对其 稹量中心的运动特点, 这对正确解释測量結果是极为 必要的。

1959年 1 月 2 日所发射的多級宇宙火箭，在历史 上首次飞进了月球区域，通过了距月球約为 5,000 公
里的地方, 抹且脫离了地球与力場而变成了太阳系的 第一个人造行星。宇宙火箭上的科学仪器和电暴的重 量計 361.3 公斤。宇宙火箭最后一級在燃料耗尽后总 重仍有 1,472 公斤。

从苏联国土上向月球方面发射火箭, 較从低緯度 地区发射要困难, 指出这一点是极有意义的。苏联国 土不能与月球軌道本面 (这个平面現时大約在北稦 $18^{\circ}$ 与南緯 $18^{\circ}$ 之間) 相交, 为了飞到月球区域就不可能利 用月球軌道平面上各种最有利的軌道。这些軌道能使 宇宙火箭在最有利的条件下实現加速飞行, 師火箭在 加速飞行段的飞行方向与它所处地点的地平面构成很 小的偏斜度。当火箭在月球軌道平面上运行时, 为了 在給定距离上接近月球所需要的控制火箭䒺統的准确 度也可以小些。这一点也是很重要的。

要知道, 一个月內各天发射宇宙火箭的意义妌不 一样。在苏联境內发射火箭, 当月球軌道在南糊 $18^{\circ}$ 附近形成最小的偏斜时, 才最为有利。距此条件太远, 就要減少有效荷載, 于是就要減少科学仪器的 数 量, 其至不能发射。选择这一天来发射宇宙火篰, 是为了 在火箭接近月球时与最佳状况最接近。

发射点愈縊接近月球軌道平面, 选择发射有利日 期的問題就愈不重献。

由于苏联制造的火箭具有高度完着的結构, 在宇 宙飞行上才能获得这样的成就。在設計和制造火篎 时，利用了苏联科学技术的最新成就。創制完善的遥 载火箭, 要經过巨大的科学研究工作, 扭要依靠我国 工业的高度发展水平。我們已制成大功率的、高效能 的、使用高热值然料的火箭发动机。創造了控制火箭 飞行的自动系統,这类系統能保证火箭在空間的稳定, 投能精确地沿加速段上的給定軌道飞行。为了将人造 卫星引入具有給定参数的軌道，或者为了实現既定目 的的宇宙飞行，对加速飞行段終点的坐标和分速計算 值必須极为精确。苏联在发射各个卫星和宇宙火箱 时, 成功地解决了上述的极其复杂的間题, 这是現代 自动化科学的卓越成就。

苏联人造地球卫星和宇宙火箭的发射, 获得了研 
究大气商层和宇宙空間方面有根本科学意义的各种結 果。

以下根据苏联科学院院长 A. H. 涅斯米掦諾夫 1959年 3 月在苏联科学院全会上所作报告的材料, 对 那些科学数据基本上已加分析的試驗結果，间述于下：

\section{地球附近和宇宙空間的宇宙輻射的研究}

近年来在研究宇宙綫方面所进行的工作，无論对 解决有关超高能量条件下的粒子相互作用的問題，或 者对解决宇宙綫起源的問題, 都取得了許多有意义的 成果。苏联物理学家和天体物理学家所发展的超新星 爆发时产生宇宙綫的理諭，将宇宙射电輻射和宇宙綫 这类現像㧤一起来了，抹提供了解决宇守綫起源的新 途径。为了証实和进一步发展有关宇宙䋐起 源 的理 論，为了扩大我們对全际和行星际空間特性的概念, 就

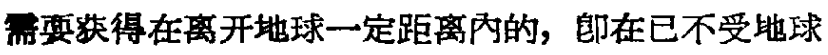
大气和地磁場影响的这一段距离內的有关原始宇宙 和粒子流的更精确的新资料。同时，还必須取得有关 粒子流强度随时間而变化以及梳子流的“化学”成分和 粒子能量蹭的资料。

从第一枇人造地球卫星进行試驗时，就向宇宙結 研究者提出了上进任务。然而, 結果是出乎意料的, 除 了原始宇宙䋐之外，在高空中曾发現了由能量較小的 粒子組成的强度极大的輻射。

苏联第二个人造地球工星，首次对地球外晨大气 的宇宙进行了长期的研究。1 1957 年 11 月 7 日莫斯 科时間 4 时 36 分, 当人造卫等正在飞过地磁緯度 $55^{\circ}$ 的区城时，記录到的輻射强度堆加了 $50 \%$ 。在同一时 閒內, 地面覌測站汼没有記录到有关强度㙁大的任何 跤的。可見, 这一效应是由于没有达到地球表面的較 小能是粒子所引起的。

在苏联第三个人造地梂卫星上装有极为灵㙁的仪 器——問嵝計数器。到目前, 对卫星在地球各地区上 室各种高度飞行时所作的大量記录作了分析。記录表 明, 无論人造卫星进入地磁緯度的北緯或南緯 $55^{\circ}$ $65^{\circ}$ 时，在所有的情况下都覌測到 $X$ 射綫强度的急剧 坆加。对已得资料的分析泼明, 仪器記录到的輻射是 由于电子隻击人造卫尾外壳而造成的。这些电子的能 量为 10 万电子伏或者小些。这些試驗还表明,在离开 地球时，所覌测到的㬏射强度是逐激增大的。

这一事实佂明，粒子不是直接来自宇宙空閒，而 是沿着磁力䋨作振㗐运动。对能量不大的带电粒子說
来, 地球磁場是一个特殊的“捕集器”。粓子在捕集器 中能够在很长时間內沿着实际上是閉合的軌道运动，

从实驗所得数据中可以看出，在与地球相交于較 $65^{\circ}$ 为高的磁緯的磁力綫上，这些条件得不到满足，[因 此接近两极的地区不受輻射影响。輻射可上据的空間 地区称为外輻射带。

1959体 1 月 2 日发射的宇宙火箭已获得了有关外 輻射带的最詳細资料。图 1 表示一个測量电离用仪器 的壊数与距地球中心距离的关系曲綫。水平軸表示以 地球牛径来度量的距离, 垂直軸則以每秒徐电子伏的 数字来表示輻射强侫。

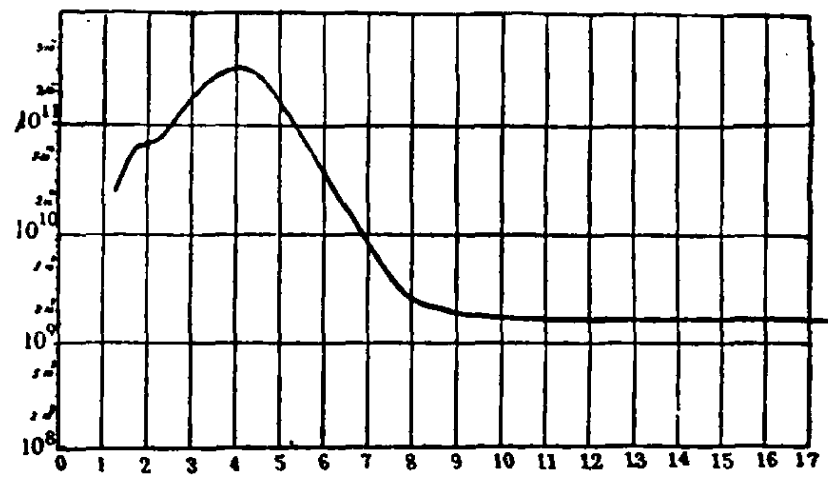

图 1 輻射強度的变化与距地球距离的关系

宇宙輻射的强度随距离地球的远近不同而有所增 长和变化,在距离地球相当于四个地球半径的时候, 它 的强度达到了頂㟯, 即增大了几百倍, 但再向外伸展 时, 强度便陡然減弱, 在相当十个地球半径的距离之 外，宇宙輻射就达到了在行星际空間的一般常态。

宇宙火箭的仪器不仅能明确外輻射带的空間分布 情况, 而且也能获得有关該带中带电粒子构成的新知 識。在最大强度区域中的电子有效能量是二万五千电 子伏左右, 而在外輻射带的边界上, 电子能量㺫将近 五万电子伏。火箭从距十个地球半径的外輻射带出来 以后, 上述仪器文十分精确地測量了行星际室間中的 原始宇宙綫以及硬电磁輻射 ( $x$ 和 $r$ 射綫)的强度。

除上述高强度的外輻射带外, 份有內輻射带。美 国人造卫星的試驗发現了在赤道区域中 1,000 公里以 上高空的輻射强度很高。

苏联第三个人造卫星所获得的有关这一現象的詳 細資料証明, 从地磁南緯 $35^{\circ}$ 到地磁北䋨 $35^{\circ}$ 的带,在 近 1,000 公里的高空中乕滿了內锚射带带电粓子。东 牛球和西半球內輻射带的下界高度是不同的: 在东半 球为 1,500 公里, 在西年球为 500 公里。这种情况是 由于磁偶极子場对地心的编移而引起的。

与外輻射带不同,在內輻射带发現了高能量粒子。 
分析第三个人造卫貹获得的資料被明，这些粓子是能 量达一亿电子伏的稹子。

图 2 的阴影区域是苏联物理学家发現的距地球很 远的外輻射带。黑色区域是高能量稹子区。

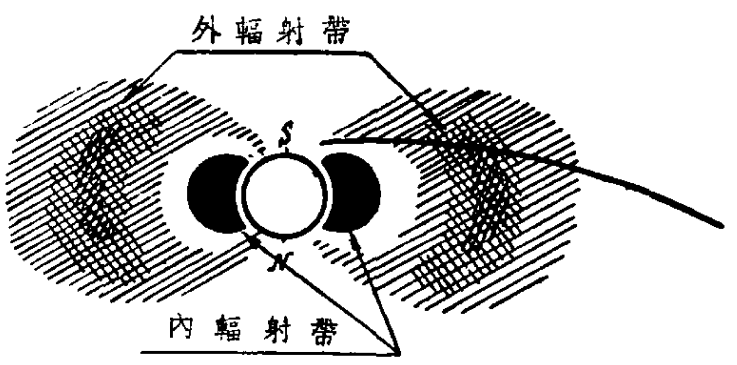

图 2 地账周围需射增強区域的形状实 綫——宇宙火箭运动的軌道

在第三个人造地球卫星和宇宙火箭上切曾作过記 录短經跡粒子的实驗。发現了这些粓子形成的很强的 粒子流。这是具有能量近一万电子伏的电子。电子通 常按接近垂直于磁力綫的方向运动。显然，这种輻射 的强度从赤道到两极逐漸增加。輻射一直伸展到相等 于几个地球牛径的距离。

发現了一种将有助于閶明大气上层中所发生的一 些过程的現像。迄今为止，关于极光还没有令人滿意 的解释。被发現的强大的粒子流可能会提供对这一現 象的答案。事实上，在地球附近一茞蕴藏着一种巨大 的能量一运动速度很高的电子。这种电子的一部分 週期地进入大气低层, 可能正是因此而形成了极光。

运动速度很高的电子在与大气上层的原子和分子 相碰撞时，便会产生 $X$ 射綫，特別是在极光分们最多 的区域內。这样，地球大气便成为 $X$ 射綫源。这种射 綫进入 100 公里以下的高空, 可起了滵度更高的大气 层的电离化。

另一部分 $X$ 射綫则射到外层空間。于是,地球(可 能还包括其它行尾）便会成为 $X$ 射綫。

地球附近粒子輻射带的性稹和起源, 是物理学家、 地䟵物理学家和天体物理学家最感兴趣的問題。从发 現这一新的現像到目前为止,只經过了很短的时間,因 此还不能就各种解释此現像的假說进行抉择。

去年夏天在莫斯科召开的国际地理物理年专門委 員会的会議上，曾經提出如下的假說：在宇宙綫的作 用下，地球和其它天体一样成为中子源。这些中子是 由于地球大气成分中的原子核被宇宙綫破坏而产生 的。这些中子是不带电荷的，它們穿过磁場，无阻碍 地远离地球。在地球附近，部分中子发生蛨变，成为 带电粒子一一电子和稹子。这些粒子的能量較小，因 而被地球磁場“鎖”在一定空間內。它們既不能进入地
球大气中, 又不能飞到行星际空間。于是, 这些带电 粒子便会长久地滞留在远离地球約数千一一数万公里 地球磁場內。在离地球这样远的地方，原子的数量极 少，因而与原子碰撞的机会讪就很少，抖且上迅粒 子的能量地就襄减得很慢。在这样长时閒中, 带电粒 子的数量愈集愈多，輻射强度怊就愈益增高。目前， 可以訓为正是这一过程产生了內輻射带中的高能质 于。

为了全面问明內輻射带的結构，必須弄清楚：是 什么方式的粒子逸出內輻射带的过程制約着內輻射带 的聜間范围。

有两种假說試图解答这个問題。一种假說䚿为， 在高空中高能质子的魀出由于 磁場的減弱而急剧加 强。另一种則䚯为, 逸出是因为在地磁緯度大于 $35^{\circ}$ 的地方磁場迅速变化。

作为解释外輻射带生成現像的最有希望的一种假 說, 䚿为它是由于来自太阳的带电粒子流的作用而发 生的。当太阳活动加剧时, 太阳射出带电粒子組成的 凝块，其中也带出了一部分太阳磁場。这些凝块的磁 場能够成为“捕集器”，在这些捕稚器中等积大量在太 阳上形成的粒子。随后，这些粒子可能被“貲入”地球 磁場所形成的“捕集器”。結果在地球附近便出現了来 自太阳的粒子。

最后，必須指出，如果原子爆炸的散落物进入商 空，他們就会在那里形成强大的带电粒子流。而因为 这些粒子的能量小，它們便会被“鎖”在磁場“捕集 器”中。因此，原子爆炸可使地球附近的宇宙空間“污 染”。

虽然自从1957年10月4日发射了第一个地球卫 星后仅仅过去了一年牛多一些，人造地球卫尾和宇宙 火箭的飞行已經作出了杰出的发現。网經被看作是司 宾的地球周围的空間，現在已經被证明是在实践和科 学上极为重要的現復发生的場所。

可以作出对于天体物理学具有根本意义的預自， 邲任何一个有着磁場的天体的周围都存在着这种粒子 輻射带。宇宙的性稹在行尾附近发生了重大的变化， 而且这种变化的范围較这些天体的大气范围大許多 倍。

根据宇宙火箭考察到的材料，行星际空間中宇宙 綫对末来的宇宙飞行者的机体将不会产生特別有害的 影响。自然, 必須說明, 这一結論是指当宇宙火箭飞 行时宇宙处于較为平靜时的状态而言的。

在地球附近的最大輻射区域, 輻射强度很大。 此，当宇宙飞船在地球附近（可能还包括其它行生》 
飞过时，应当估計到运动速度很高的粒子呩吉飞船船 身的危险。这种車吉可能 給生物机体带来放射性疾 病。

对这种輻射的防护是可能的嗎？已获得的材料証 明，在外輻射带，防护是能够作到的，尽管为了防护 需要增加飞船本身的重量。同时，在粒子能量很高的 內輻射带中，有效的防护設备需要更多地增加飞船的 重量，因此，末来宇宙飞行者所乘坐的火箭的弹道应 当这样确定: 在輻射带中, 尤其是在內輻射带中飞船 飞行的时間不能过久。

在第三个人造地球卫星上，装置着用来研究宇宙 䄉中超重原子核仔在問題的仪器。契連科夫探測器曾 記录到每个核子的动能超过 3 亿电子伏的原子核。这 种仪器是用来記录两类原子核的：电荷大于 15 的和 电荷大于 35 的原子核。对于数据的分析表明，每分鈡 平均有一个电荷大于 15 的粒子通过仪器。在元昼夜 中，用来記录較重原子核的波道仅仅有一次动作。这 就是說，应当培为，重原子核流是很弱的。这一事实 对于进一步研究宇宙綫起源的理論具有重大的意义。

\section{对高层大气的研究}

发射卫星和火箭所提出的最主要任务之一，是要 研究高层大气的結构。高层大气的范围,是从大約 200 公里的高度一直延伸到大气的外䊏。研究高层大气是 与解决一系列困难問題关联着的。

問題之一是高层大气的热平衡間題。在 200 公里 的高度, 周围环境的溫度等于 $800-1,000^{\circ} \mathrm{C}$, 以后增 高到 $2,000-3,000^{\circ} \mathrm{C}$ 。由于溫度的数值很高, 所以大

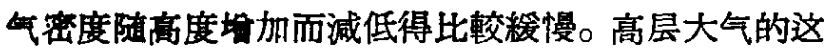
样高的热度，究竟是从何而来呢？我們用前面提到的 火箭和卫攵所得的关于这个問題的一些数据, 得出了 新的結果。

在試图解释大气电离平衡, 師在自由电子和离子 的发生与它們的中和之間达到平衡的过程这个問題的 时候，曾遇到了不少的困难。如果从以下覌点出发， 即放为中和过程是通过电子与正离子借助于放出光量 子的能量而进行的, 那末, 实驗結果与理論計算要相 差千倍至万倍之多。

事实表明，当有其他粒子参加进去的时候，这个 过程的进行是极其微妙的，这些粒子缘催化剂一样， 大大地加速了这个过程。

为了調节电子的中和过程，这些粒子只占中性粒
子或自由电子数的万分之一或十万分之一就足够了。 例如，一氧化的正离子就可以作为这种催化剂，这 种离子是在 200 公里以上的高度，借助于安数在第三 个苏联人造地球卫量上的頎譜仪而发現的。

这些研究对于实践所起的作用是巨大的．大宾䂙 很熟悉, 正是由于电离层的电磁性质，无綫电波才能 传播到很远的距离。

这里可以指出一个有趣的現像，这个現虽然在 过去也知道，但在覌測苏联地球卫尾信号时表現得更 为显著。这个現稣称之为对踱效应, 具体表現为: 在 地球上与发送电台处于对踱地位的地方所收到的信 号, 其强度有所增大。根据第一个工星的无縛电信-号 在南极和平村接收結果的記录，可以看出卫星正在和 平村上空和在和平村的对踱点时的 20 兆赫无綫电信号 的接收情况。你这样的情况，郎在电离层中长时關保 在着使无綫电波“流向”地球的对踱区的有利条件，是 具有很大意义的 $c$

但是我們也知道电离层对于实驗的不利之处。在 研究用无綫电方法控制末来的星际“飞船的时候, 电离 层的影响可能在决定飞飞船的座标、速度等等中引起誤 差。为了排除这种誤差, 必须了解电离层的結构。监 于以上所述, 苏联科学家近来䛈得的科学結果, 其意 义就更为明罡。

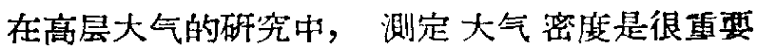
的。在第一个苏联卫星发射之前，所获得的确实可靠 的数据仅限于 $150-180$ 公里的高度。及至 250 公里 的高度, 所用各种方法获得的数据, 彼此之間矛盾很 大；而关于 300-350 公里以上的大气密度，則是一 无所知。

苏联科学家用各种方法对大气櫁度作了研究。根 据卫星在繞地球运轉时，由它們的制动所引起的时間 变化, 可以十分准确地在軌道的近地点測定其与大气 密度成比例的数值。

第三个卫星上會装設了特种类㕵的气压計，借以 对 225-500 公里高度范围的空气滵度作了測量。

此外，根据第一和第二个工星的无綫电信号所測 定的电子浓度，对 $320-1,000$ 公里高庭处高层大气 密度的平均变化作了計算。也曾利用了一种独特的对 鈵云的扩散非行覌察的方法, 这种鈵云是发射高空火 箭后在430公里高度喷出的。根据鈵云的扩散特性, 在 扩散理論的基础上計算出了上述高度的大气密度。类 似的鈵云，后来在苏联宇宙火箭上形成人造慧星时进 一步加以利用。

密度剆定的結果示于图 3。在这个图表中，根据 
現有資料把密度換算成为每立方厘米的中性粒子数。

这些硎究的結果是一致的, 根据这些結果, 第一 次准确可靠地确定了 600-800 公里高度的大气滵度。 它們也証明了在卫星发射前存在着的一系列概念，有 不少是錯誤的。根据这些数据，作出了大气模型。通 过对卫星制动的經常覌测，揭示了緯度的和昼夜的密 度变化。根据卫蛋的制动，也获得了一些关于高层大 气溫度的数据。在 228 和 368 公里的高空中, 溫度在 $800-1500^{\circ} \mathrm{C}$ 范围內变化。

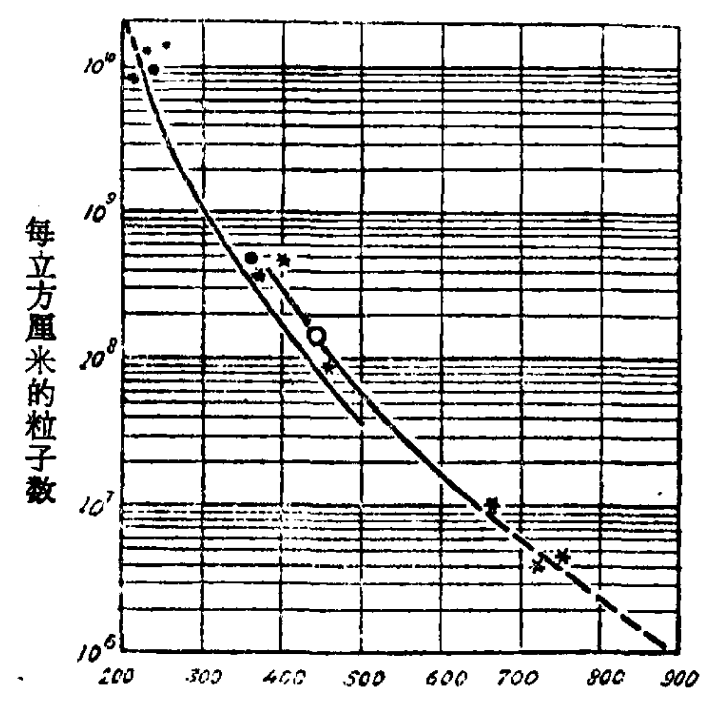

高 度 (公里)

国 3 用各种方法获得的中性粒子密度随着高度变 化的曲綫。

图中○为根据对苏联卫星制动的研究而測定密度的 結果。

○为根据鈎云扩散而測定密度的結果。

*为根据对苏联及美国卫星制动的研究，在世 界交献中常用的数据。

实綫相当于用装在第三个苏联卫星上的气压 計和根据第一个苏联卫星的无綫电信号而获 得的結果。

在 226-1000 公里的高空中，會利用壮置在第三 个卫尾上的稹譜仪获得厂大量表明电离层化学成分的 正离子质譜。在原子质量数 6-48 的范围內进行了測 量。质譜仪的測量結果，表明貭量数 16 的离子为数 最多。从 226 公里到至少 800 公里的高空中，原子氧 是基本的气体成分，电离层就是由于它而产生的。

除了原子武的离子以外，也記录到原子氮的离 子; 阁时还发現了 28 和 30 原子量单位的重粒子。盾 量为 30 的离子可能就是一氯化氮。按上面的說法, 在 350 公里高空发現这些粒子, 是十分有意义的事, 待 将来确定这些事实后, 可能有助于解决高层大气电离 平衡的問題。
原子氮与原子氧的相对含量, 可随高度和地理緯 度从 $1 \%$ 改变到 $10 \%$, 牛且也，可以随时間而变化。一 氧化㓱和分子管重离子的相对含量, 可随着高度的 增加而急剧降低。在 230 公里的高空中，一化露的 离子数与原子氧的比为 $25-40 \%$ 。

已获得的大量資料証明，电离层成分与地理緯度 具有一定的关系。特別在 226-260 公里的高空中, 在 北緯約 $60^{\circ}$ 区域內探測到原子燚离子的含量在急剧增 加。

由盾譜仪获得的瓷料說明，卫星在白夫的目电位 平均約为 5 伏特。

在測定带电粒子浓度方面，也获 得了重要的結 果。利用装設在地面上的各种无綫电仪器, 只能測到 电离层主最大电离高度以下的电子浓度分仍，在各种 不同的情况下，电离层的主最大高度約在 300-400 公里之間变化。在主最大电离高度以上, 电子浓度的 分们情况，在第一个卫星发射前，始終还是个謎，虽 然有些科学家, 特別是根据美国火箭探室的数据, 管 經訩为在主最大电离高度以外的高空, 电子浓度迅速 下降了。

苏联已采用各种方法研究了这个問題。垷在, 对 第一个卫星无綫电信号軌跡的分布，已經測定主最大 电离高度以上在 320 - 650 公里高的电 离层电子浓度 的平均分布。

1958 年 2 月 21 日, 直发射的苏联地球物理火 箭第一次直接測量了在 470 公里高空电子浓度的分布 情况，在 1958 年发射其他火箭时所获得了类似的数 据。

在第三个卫星上，首先利用直接法測量了在 900 $-1,000$ 公里高空中卫星軌道上的正离子浓度。測量 是用所謂离子捕集器进行的, 抹已获得大量的实驗数 据。由于高层大气的正离子浓度接近于电子浓度, 因 此这些实驗可以提供电子浓度的情报。利用捕集器进 行的試驗还能測量卫星相对于周围介稹的自电位, 筫 电位在太阳炤射到的軌道区域等于 $1-7$ 伏特。

快速电子作用于卫玍的效梁, 同样可以闹明卫尾 的負电位值, 这种快速电子的能量大大超过大气粒子 的平均能量。

关于主最大电离高度以上的高空中带电粒子浓度 的研究結果, 可参閱图 4 和图 5 。主最大电离高度以 上电子浓度的下降比在低电离层电子浓度的增加要慢 一些。

根据这些数据向較大的高度上外推可以設想，在 $2,000--3,000$ 公里的高空中电子浓度应达到每立方 
男米不少于数百个电子的值, 也就是說, 等于行星际 气体的平均密度假定值。現在看来, 地球大气至少伸 展到 2,000-3,000 公里, 从而否定了过去的大气只 达到 1,000 公里左右高空的說法。

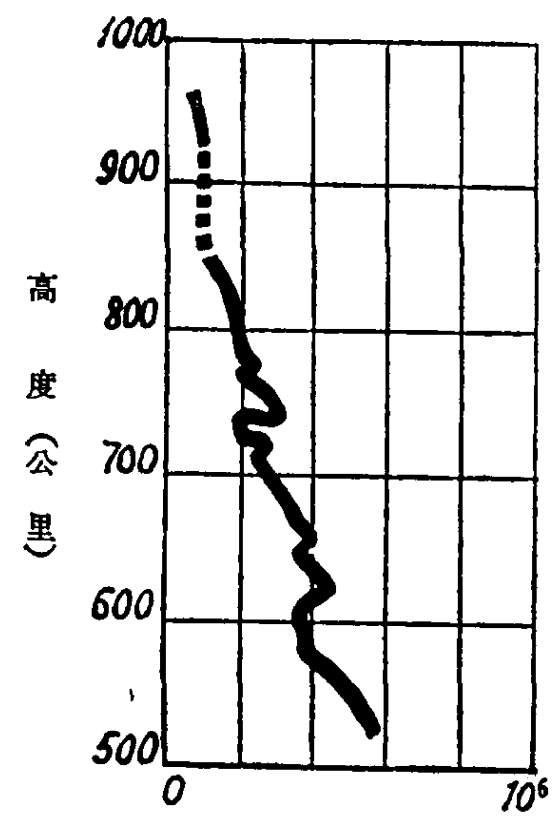

离子数 / 立方暒米

图 41958 年 5 月19日在莫斯科时間11时左右由装 在第三个苏联人造地球卫星上的禹子捕集器所获得 的正离子浓度与高度 变化的曲綫。实程是測量数 据，感絊是应用內插法得到的数值。

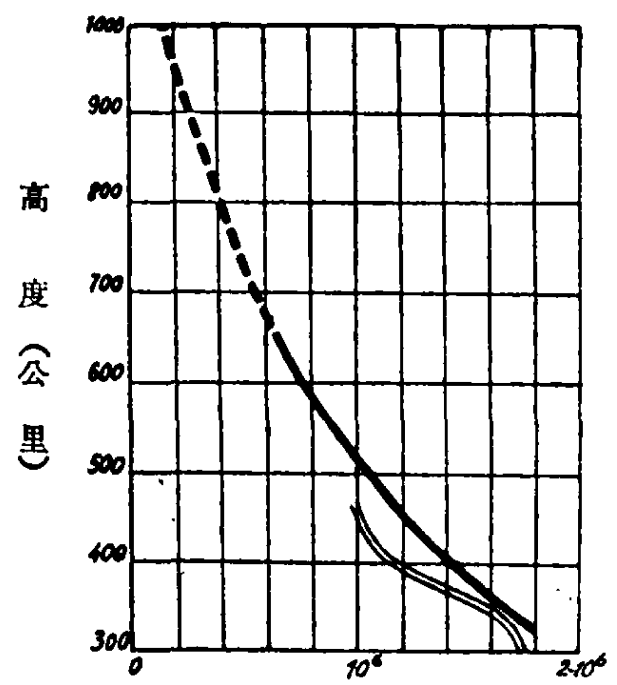

电子数/立方風米

图 51957 年 10 月 $5-8$ 日 7 时 40 分一 9 时 40 分 根据第一个苏联人造地球卫星无䋐电信号記录整理 后的电子浓度与高度变化的曲䋐(实綫), 以及 1958 年 2 月 21 日 11 时 40 分发射高空地球物理火箭記 录到的曲綫(双綫)。虚綫表示应用外推法得到的数 值。

\section{行星际气体的研究}

苏联宇宙火箭初次进行了有关酸接研究行星际物 盾的气体成分的試驗。

装置在宇宙火箭中的 仪器 是为了第一阶段的研 究，郎直 接 覌 測地球与月球之間的行星际离子化气 体。这种仪器有四个三极的正离子(貭子)捕集器, 捕 集器的网状壳充电到对容器外壳具有各种电位。这样 的仪器不可能充分頋及到在进行測量时壳对周围介顿 的电位影响。因此, 利用这些仪器不能精确測量正离 子的浓度(精确測量将在今后才能办到)，而根据記冰 下来的电流数值仅可获得初步估計。这些捕集器的收 集器电路中由正离子所产生的电流，可以表征火箭路 径上漓子化气体粒子的浓度。

目前，試驗的紹果还在进行整理。但是現在已經 能够提出某些具有重大意义的資料。

根据初步瓷料，在 1,500 公里高空，在不受日光 照射的区域里的大气，每立方厘米中正离子的浓度为 1000 个; 在 2,000 公里的高空 (同样是在未受日光炤 射的区域內)，正离子的浓度約为上进的 2/3。距离地 球表面 21,000-22,000 众里的地方，正离子的浓度 約等于 2,000 公里高空处阴影区域內的离子浓度。在 距离为 11-15 万公里上, 由捕集器 所記录的电流可 以算出在火箭經过的路径上，正离子的浓度的为每的。 方厘米 300-400 个。

这一实驗置，初步可估計出行星际空間离子化 体的浓度，这些数据不是根据間接的可作不同解释 的地面观測数据, 而是根据直接測量得出的:

\section{地磁研究}

关于外层空間中的地磁場的知識，对于活多地磁 学的問題来說, 是具有重大意义的。根据装在苏联第 三个人造地球卫星上的地磁仪所取得的地磁記录图的 分析結果，可以作出在地球物理学上有基本意义的 系列結論。

根据关于地磁日变化的現代概念，可以期堭在卫 星两次沿同一地球弧行行的时候，对磁扰現像进行 最有效的探究，其中一次在磁靜时，另一次則在磁扰 时，但要尽可能在同一天中的不阔时刻进行。在上述 情况下測得磁場的二个数值之差，应跬与外部电流系 
統效应或者与它的一部分效应相等。而且对于地球的 白昼和黑夜部分上的相应軌道綫段来說, 此差俻还应 部具有相反的符易, 因为正的磁扰旅浴和自的磁扰旋 涡是同时存在的, 而卫星經过的时間則有 12-15分鈡。

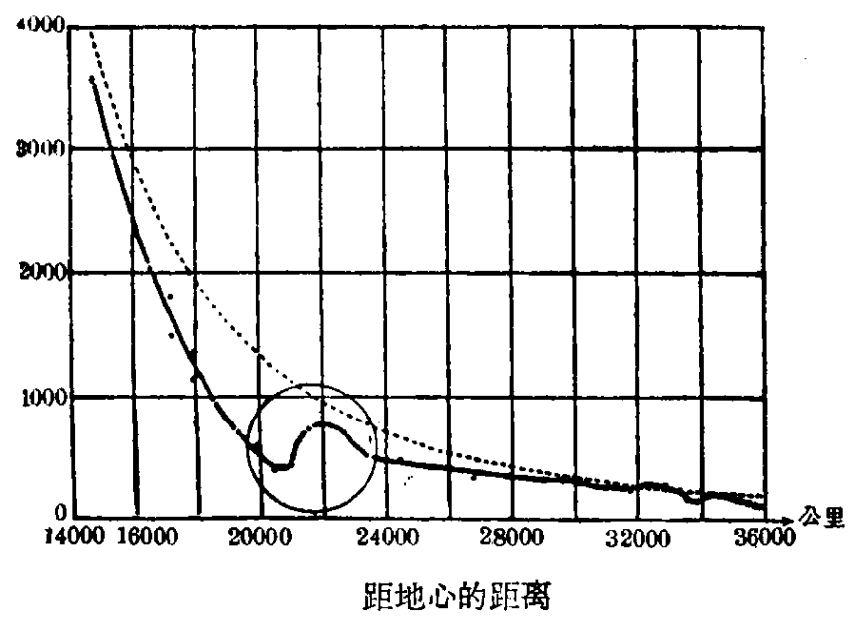

图 6 地磁昜強度随高度的变化曲䋐。图中用图圈 划出的是苏联宇宙火箭在飞行中发現的外电离层电 流环的效应。秋座标为地磁場強度, 单位为伽瑪。地 磁場強度的部算值用黑点表示。实綫为測量数据。

第三顆苏联卫䒜所作的磁場㸴究, 确切地証实了 裂引起磁扰的电离层的变化。在分析从工攵上收到的 磁記录同时，发現了 20 次磁場快速变化 (5-8 秒)的

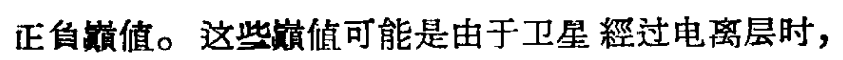
电离层中的电流系統具有地区性的不均匀所致。

这些結果对于建立电离层的物理模型以及磁扰的 定量理論来說，有着重大的意义。

在对地磁場常数进行研究的时候, 又取得了新的 有价值的数据。最重要的数据是当卫售在东西伯利亚 伳界性地磁异常区域, 郎所胭“亚洲最大地磁場”区域 的上空飞行时取得的。分析磁記录綫图抹将它与沿 卫星飞行路地面的地磁图作一比較, 可知地磁异 常随高度的降低是緩慢的。这一事实在解决关于世界 性地磁异常源的潛藏深度, 以及关于地磁場的性稹和 結构的問題上有着重大的意义。根据它就可以作出关 于东西伯利亚地磁异常具有深源的結論。

利用宇宙火箭测量地磁場所得結果, 按其本身的 意义来說, 是特別重要的。磁場强度的測量檤与理論 計算值之間的差异，在距地心約两个地球牛径处就非 常明显了, 往后郎逐漸算大(图 6)。

实际的地磁場强度(較理論計算所得的)递降得快 一些, 大約在距地心 20,800 公里处达到最小值, 約 为 400 伽瑪, 亦師为地面磁場强度的百分之一左右。 此后, 磁場强度及見回昇, 在 22,000 公里处达到最大
值, 800 伽瑪, 然后就再次下落。地磁場的这种变化, 只能根据这样的假定来加以解释, 郎火箭在 20,00021,000 公里上空遇到了电流。因此, 宇宙火箭上測得 的磁变化就证明了外电离层电流系統是的确存在的。

这一事实对于磁暴理論和极光理論来說, 有着根 本的意义，尤其是在批判地評价目前解释此等現像的 假說的时候。

另一个重要的事件是, 电流层效应是在完全磁静 日出現的, 而最相近的大磁扰（磁暴）发生在几乎一 个月之前。在磁暴最剧烈期間发生的电离层外电流采 統, 显然可以在一个很长的时問內継績存在下去。

探測結果无疑地是地磁学以及地球物理学和等离 子区物理学等有关学科的理論研究疽料。探清磁場最 大值与带电粒子带之間的关䒺，是很重要的。

装在第三个卫星上的地磁仪, 除了測量地磁場以 外, 还能够取得关于卫星在太空中的方向的数据, 和 研究它的相对于重心的运动。这些数据在解释卫星上 同时进行的大部分探測的結果时是必需的。

\section{微 流星}

为了記录微流星，在苏联第三个人造地球卫星上 淩有一架可以記录微粒撞古次数及其能量的仪器。它 是根据传感器所受冲量而确定, 这冲量就是当流星在 其表面冲击吋所損耗的。

根据微流星的能量与冲量之間的理論关系, 应假 定粒子的平均速度为每秒 40 公里, 則在該仪器的工作 期間內, 記录到了稹量 80 亿分之一到 2 亿分之一克、 能量由 1 万尔格到 10 万尔格数显級的粒子的撞古。

正如在国际地球物理年第五次全会上所报告的那 样，1958 年 5 月 15 日記录到的庫古次数較以后記 录 到的为多。这一天所記录到的数字是每平方公尺每秒 4-11 次。和 5 月 15 日相比, 5 月 16 和 17 日揞击次 数降低到 4 千分之一, 其后又降低到 5 万分之一, 最 后降低到 60 万分之一。

由传感器記录下来的冲量与粒子能量之間的比例 系数的数虽, 最后将田实驗(用模型方法)加以测定。

根据用宇宙火箭所作的实驗可以作出結論, 质盖 約为 10 亿分之一克的粒子, 可能每数小时与火箭表面 磁撞一次。

正如从苏联第三个人造工星和宇宙火箭的測量桔 果中可以看到的那样，流星或者微流星的危险性是很 小的。 


\section{生物学的研究}

現在已出現了一个新的科学部門——宇宙 生物 学。它的主要任务之一是保障人在宇宙空間飞行中的 安全。

在火箭上进行的研究表明，实驗动物在飞行中完 全可以經受不同性质的因素对机体的作用。由現在积 霜的资料可以得出結論：在近似宇宙飞行的条件下， 实騟动物的主咏生理机能状态没有 发生明 显的失調。 大概最复杂的間题是保障动物在地球上着陸。

現在在这方面得到了人所共知的成就。实驗动物 可以从数百公里的高室安至着陸。人造地球卫星可以 提供更多的可能性, 因为, 从生物学覌点看来, 人造 地球卫星上的条件是与宇宙飞行的条件最近似的。

鲜細分析由第二个人造地球卫星得到的科学情 报，可以取得一采列新的有意义的盗料，首先是有关 长期失重作用的资料。

当失耍时，没有发現动物的植物性机能有任何不 良的反应, 这个情况是极其重要的。同时, 动物没有 对运动表現出显著的不安。

图 7 为小狗“萊伊卡”在卫星飞行的不同阶段內心 琏活动变化的动态。图中的曲綫表示心脏收縮頻率的 变化。不难看出，卫㚅被引入仇道时的加速度、簏动 和噪䕗, 引起了心悸頻率的急剧增大, 而达到最大的 数值。在失重的条件下脉博頻率逐潮恢复原状。

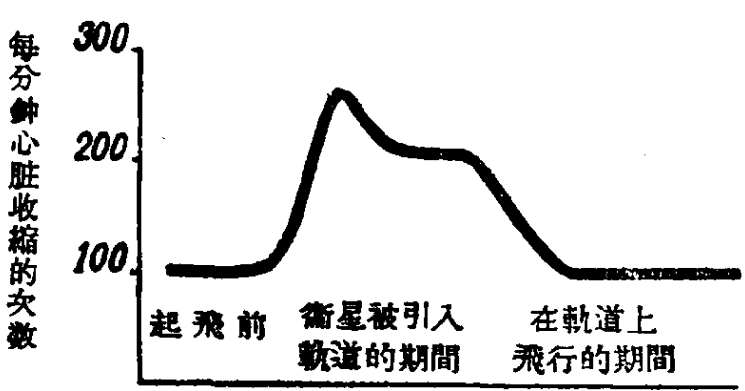

图 7 在不同飞行价段內小狗“萊伊卡”心肚收縮頻率曲䋐 心电图(图 8)的鋸齿形波、呼吸运动和活动力,沟 有表現明显的异常。

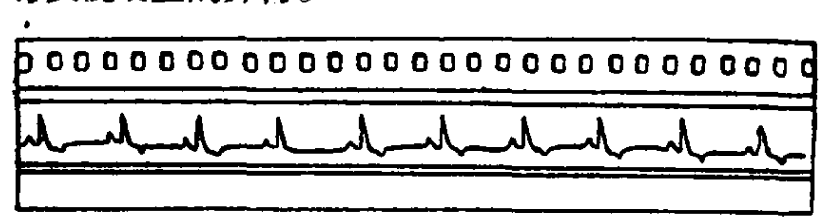

图 8 在第二个苏联人造地球卫星上所記录的小狗 “萊伊卡”在失重情况下的心电图
心电图的間隔的改变一般是符合心脏收縮頻率的 改变的。这証明心肌传导机能没受摜䔄

总之，已得的結果明显地表明，高敉动物完全可 以經受接近宇宙飞行的条件，

\section{宇宙飞行发展的基本方向}

苏联的科学家、設計师、工程师、工人、实駿技 师在发射人造地球卫星和宇宙火箭（利用它們使直接 哳究宇笛空間成为現实) 当中作出了卓越的貢献。我 們的数学家、力学家、各种专业的物理器寀也有很大

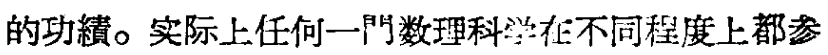
加了解决研究宇宙空間这一垂大間題。这种科学和技 术的綜合带来了显著的效果, 由此, 現在已可預見到 宇宙飞行将来发展的道路。

宇宙飞行的发展在不久的将来将朝着許多方向前 进。人造卫星在地球周围飞行是一个方向, 号一方向 是解决飞向月球和探明有关月球的問題。第三个力向 就是研究太阳柔队的空間、太阳系的行星和“向其他 行星。

人造卫星可以解决广泛的科学和应用問題。苏联 的前几个人造卫星已可用来进行大量的研究工作, 可 用以研究地球大气外层以及与它毗連的宇宙室間区域 中的許多現象。

无論在扩大科学研究范围方面, 或在用人造卫.星. 解决純应用問題方面，都得进一步发展工星的工作，

需要制造用一定方法使 其在空 間 定向 的人造卫 星。䚺多科学問题的解决都需要定向。諸如, 对于許 多与太阳有关的研究，都希穜人造卫坣能对太阳定向。 对和地球及大气有关的呼究来說, 最适宜的定向是使 卫星軸之一向㔨地球，而另一軸时沿其軌道与运动的 方向一致。对于天体物理研究来說，卫星最好的定向 是卫星的位䈯与相对不动的恰星保持不变。

最重要的阶段是由人在卫尾中非行駕駛，为此需 要解决大量的极其复杂的間题，即保障安全和造成必 要的条件，以解决人在起飞和降落时的超重作用下及 沿軌道“行行时失重情况，下的生命活动問題。在第二:个 苏联人造地球卫星上用試驗动物所做的实䮑，得出了 关于宇宙飞行条件对生物有机体作用的科学数据, 这 是在这方面得到的第一批寅要的結果。

多次发表了关于利用特殊的卫星系統来轉播电視 的可能性的概念，这样就可以保障超短波段的远距离 传送，而不用再設无綫电接力站綫网。 
利用卫星可以組成固定的覌測站，以覌測太阳的 微粒輻射, 它还可以保証大气外层发生的最重要現像 的預报。

如同在航空的初期不能預言今天航空的多方面运 用和进展一样, 現在还很难預言卫星在科学和实践方 面的全部用途。

宇宙飞行发展的第二个方向則是一些和探明月球 有关的問題。苏联宇宙火箭表明，飞向月球和太阳系 空間的时代已經开始。

可以想権在今后, 可能还不是太近的将来, 人可 以飞到月球上着陸, 然后司到地球上来。在月球表面 着陸設备的問題十分复杂。由月球起飞和回到地球上 来，困难也汼不很小。

在更远的将来，在探測月球的过程中，可以設想 在月球上建立特殊的同地球上难以到达的地区（如极 地）一样的科学站。同时还必須指出这件事的特殊复 杂性。只有在火䈤技术大大提高和解决了大量的科学 和技术問題之后, 这件事才可能成为現实。但也說不 定在今天看来还完全是奵想和不能实現的計划, 会比 原来想鲳的实現得早得多。

宇宙飞行发展中具有独立方向的第三个問題, 是 和研究太阳系內的室間以及太阳系行星有关的問題。

在太阳系范围內飞行的目的之一，是直接研究行 星际介质。利用科学仪器探測行星际空間, 可以測定 离太阳不同距离上的行星际气体的窑度, 測定行星际 气体的化学成分, 得出太阳系不同区域中有关宇宙輻 射强度分布及其成分的极为重堻的数据, 能够研究各 种类型的太阳輻射, 研究太阳磁場及其对行星际介稹 中各种現像的影响。

研突太阳系的行星, 首先是金星和火星, 具有特 的意义。分析表明, 在一定的时間內, 当地球和行 星的相互位着使得火箭加速所需的能量消耗可減至最 低限度时，向太阳系各行星飞行是最适宜的。

向行星发射載有自动仪器的火箭, 可以研究各行 星的磁場,輻射带, 扑能得知它們表面的具体形状。另 外, 还可以研究各行星的大气, 測定其䎹度、化学成 分、电度，以及研究各行尾表面的結构及其溫度。 取后，研究其它行星上生命形态的前景是十分誘人的。
人飞上行星是未来的事情, 然而这个日子无疑是会到 来的。

宇宙飞行的发展向科学和技术提出了䚺多最复杂 的科学研究和工程設計方面的課題。

为了測定火箭轨道的参数, 把仪器測量的結果传 送到地球上扞报导仪器的工作情况以及传途地球上的 命令等等, 最重要的問題是无綫电通訊。在发射第一 个苏联宇宙火箭时,在历史上第一次在距地球約 50 万 公里处实現了无綫电涌訊。

在太阳系范围內飞行时, 需要在数干万和数亿公 里的距离上进行无總电通訊和传送訊号。因此, 制造 輕便的、小型的和极其經济的无綫电仪器, 以及地面 用的发射功零特强和灵敏性极高的接收置就具有特 殊意义。

宇宙火箭上的全部仪器不仅应是最輕便和經济 的, 而且还应該是非常可靠的, 能够在数月, 甚至几 年內持績工作。对于在太阳系內飞行而言一定姴有这 样数量級的持續工作时間,如果想想行星迴轉的周期, 那么在这方面就不会有任何奇怪之感了。仪器在宇宙 間工作的特点也取决于宇宙輻射的作用和宇宙飞船周 围的高度真空。重要的是保持仪器正常工作所必需的 溫度。宇宙飞行的严重問題之一是防护流星的撞击。

有关計算宇宙飞船运动的問題, 是天体力学的一 个新方向。在天文学史中第一次計算了人造天体的运 动, 其中也包括能够主动影响本身运动参数的不平凡 的天体。研究这些人造天体的运动, 可以取得有关太 阳系的天文常数和万有引力場的新数据。我們将亲眼 看到可以被称为天文学新的一章, 師实驗天体力学的 誕生。

发展宇宙飞行(人类活动全新的部分)方面的进步 向科学和技术提出了极高的要求: 运用一切最新的和 最先进的科学技术成就, 創造新的科学技术方向。

不容置疑，为苏共第21次代表大会制訂的在我国 建設共产主义社会的伟大綱領所鼓舞的 苏联 科学家、 設計师、工程师、工人，将光荣地解决这一最有意义 的問題，我們将是苏联在掌握宇宙室間方面的新的烟 煌成就的見証人。

（原交载 1959 年 7 月 15 日苏联“置理报”） 